\title{
BIJECTIONS FOR HOOK PAIR IDENTITIES
}

\author{
C. Krattenthaler ${ }^{\dagger}$ \\ Institut für Mathematik der Universität Wien, \\ Strudlhofgasse 4, A-1090 Wien, Austria. \\ e-mail: KRATT@Ap.Univie.Ac.At \\ WWW: http://radon.mat.univie.ac.at/People/kratt \\ Submitted: March 4, 2000; Accepted: April 10, 2000.
}

\begin{abstract}
Short, bijective proofs of identities for multisets of 'hook pairs' (arm-leg pairs) of the cells of certain diagrams are given. These hook pair identities were originally found by Regev.
\end{abstract}

1. Introduction. In their work [7] on asymptotic analysis of degrees of sequences of symmetric group characters, Regev and Vershik obtained some hook formulas, which led them to conjecture surprising identities for multisets of hooks. These identities were shortly thereafter proved independently by Bessenrodt [1], Janson [2], and Regev and Zeilberger [8]. Another such identity was added by Postnikov and Regev [4]. Moving one step ahead, Regev [5] observed that in fact all these identities are not only true as identities for multisets of hooks, but even as identities for multisets of the corresponding arm-leg pairs. He called the latter (and we follow this convention) "hook pairs." As is shown in [5], these identities imply several nice formulas for special evaluations of Schur and Jack polynomials. All the aforementioned identities feature hooks and arm-leg pairs of regions which are built out of (nonshifted) Ferrers diagrams. Finally, in [6], Regev provided similar identities for regions resulting from shifted diagrams.

Regev proves his multiset identities in $[5,6]$ by inductive arguments. (The proofs in $[2,4,8]$ are also inductive, only Bessenrodt's argument in [1] is combinatorial.) The purpose of this paper is to provide short, bijective proofs of all these identities. In fact, what I am going to demonstrate is that there is just one "master bijection" out of which all the identities result straightforwardly.

In the next section we provide all the relevant definitions and formulate, in Theorems 1-3, three key identities from [5, 6], which straightforwardly imply all other multiset identities in these two papers (and, thus, all the multiset identities in [1, $2,4,7,8]$ ). In the subsequent Section 3, we present our "master bijection," which

1991 Mathematics Subject Classification. Primary 05A15; Secondary 05A19 05E10.

Key words and phrases. hook pairs, hook-content formulas, arm, leg, multiset identities.

$\dagger$ Research partially supported by the Austrian Science Foundation FWF, grant P13190-MAT

Typeset by $\mathcal{A}_{\mathcal{M}} \mathcal{S}-\mathrm{T}_{\mathrm{E}} \mathrm{X}$ 
immediately implies the first of these identities, Theorem 1 . The resulting proofs of Theorems 2 and 3 are then given in Sections 4 and 5 .

2. Identities for multisets of hook pairs. We recall some basic partition terminology (cf. [3, Ch. I, Sec. 1]). A partition is a sequence $\mu=\left(\mu_{1}, \mu_{2}, \ldots, \mu_{\ell}\right)$ of positive integers, arranged in weakly decreasing order. We identify a partition $\mu$ with its Ferrers diagram, which is an array of cells with $\ell$ left-justified rows and $\mu_{i}$ cells in row $i$. To each cell, $c$ say, we associate two numbers, the arm length and the leg length of $c$. The arm length $a(c)$ of $c$ is the number of cells which are (strictly) to the right of $c$ and in the same row as $c$. Similarly, the leg length $l(c)$ of $c$ is the number of cells which are (strictly) below of $c$ and in the same column as $c$. We call the arm-leg pair $(a(c), l(c))$ of a cell $c$ the hook pair of $c$. For example, Figure 1 shows the Ferrers diagram $(5,3,3,1)$. The marked cell has arm length 3 and leg length 2 , thus, the corresponding hook pair being $(3,2)$. We adopt the convention of writing $a^{b}$ for a part $a$ of a partition which occurs $b$ times, so that the partition $(5,3,3,1)$ could also be written as $\left(5,3^{2}, 1\right)$.

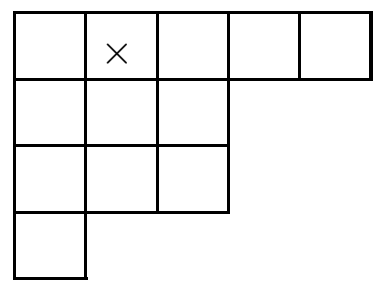

Figure 1

Now let the partition $\mu=\left(\mu_{1}, \mu_{2}, \ldots, \mu_{\ell}\right)$ be given, and choose $k$ and $n$ such that $\mu$ fits in the $n \times k$ rectangular partition $\left(k^{n}\right)$, i.e., $k \geq \mu_{1}$ and $n \geq \ell$. Let us denote the rectangle $\left(k^{n}\right)$ by $R_{n, k}$. Under these assumptions, we form two skew diagrams, $S R_{n, k}(\mu)$ and $S R_{n, k}(\widetilde{\mu})$. The skew diagram $S R_{n, k}(\mu)$ is formed by starting with the $k \times n$ rectangle $R_{n, k}$, removing a copy of the partition $\mu$ from the top-left corner of $R_{n, k}$, and then gluing a copy of $\mu$ to the right of $R_{n, k}$ so that the first row of this copy of $\mu$ is glued to the first row of $R_{n, k}$. See Figure 2.b for an example with $k=6$, $n=4, \mu=(5,2,1)$. The skew diagram $S R_{n, k}(\widetilde{\mu})$ is formed in a similar way. Again one starts with the $k \times n$ rectangle $R_{n, k}$. But now a copy of the partition $\mu$ which is rotated by $180^{\circ}$ is removed from the bottom-right corner of $R_{n, k}$, and then a copy of $\mu$ which is rotated by $180^{\circ}$ is glued to the left of $R_{n, k}$ so that the last row of this rotated copy of $\mu$ is glued to the last row of $R_{n, k}$. See Figure 2.c for an example with $k=6, n=4, \mu=(5,2,1)$.

Now we are ready to state the first of Regev's hook pair identities [5, Theorem 2].

Theorem 1. The multiset of hook pairs of the cells of $S R_{n, k}(\mu)$ is identical with the multiset of hook pairs of the cells of $S R_{n, k}(\widetilde{\mu})$.

Still given a partition $\mu=\left(\mu_{1}, \mu_{2}, \ldots, \mu_{\ell}\right)$ and integers $k$ and $n$ such that $k \geq \mu_{1}$ and $n \geq \ell$, we form another skew diagram, $S Q(n, k, \mu)$, by starting again with the 


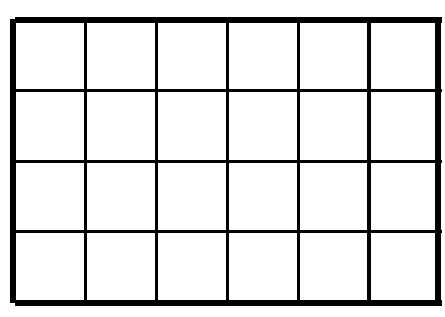

a. The rectangle $R_{4,6}$

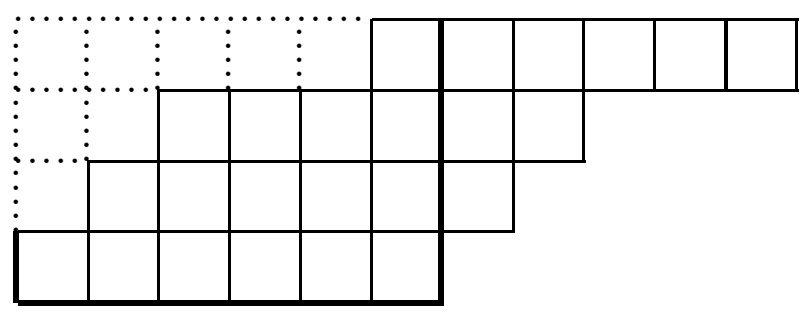

b. The skew diagram $S R_{4,6}((5,2,1))$

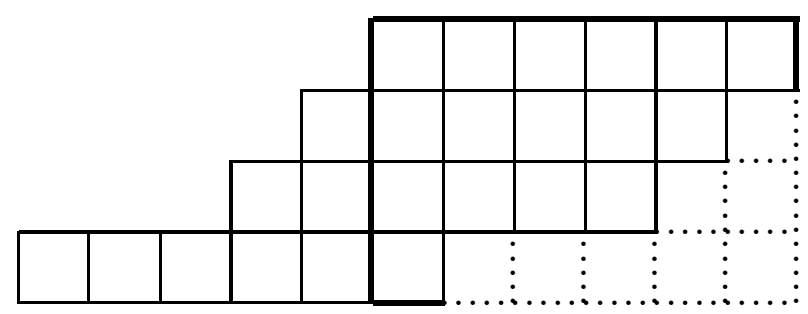

c. The skew diagram $S R_{4,6}(\widetilde{(5,2,1)})$

Figure 2

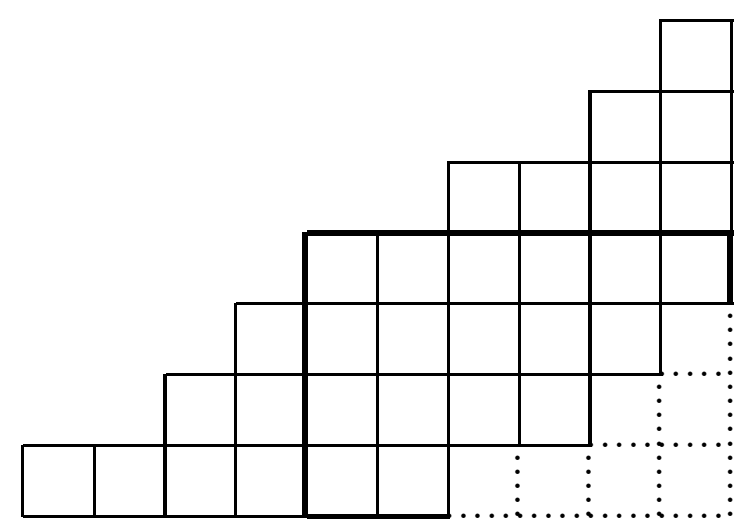

The skew diagram $S Q(4,6,(4,2,1))$

Figure 3

$n \times k$ rectangle $R_{n, k}$, removing a copy of $\mu$ which is rotated by $180^{\circ}$ from the bottomright corner of $R_{n, k}$, gluing such a rotated copy of $\mu$ to the left of $R_{n, k}$ in the same way as before when we formed $S R_{n, k}(\widetilde{\mu})$, and finally gluing a rotated (by $180^{\circ}$ ) copy of $\mu$ to the top of $R_{n, k}$ so that the last column of this rotated copy of $\mu$ is glued to the last column of $R_{n, k}$. See Figure 3 for an example with $k=6, n=4, \mu=(4,2,1)$.

The second of Regev's hook pair identities [5, Theorem 1.(a)] reads as follows. 
Theorem 2. The multiset of hook pairs of the cells of $S Q(n, k, \mu)$ is equal to the union of the multiset of hook pairs of the cells of $R_{n, k}$ and the multiset of hook pairs of the cells of $\mu$.
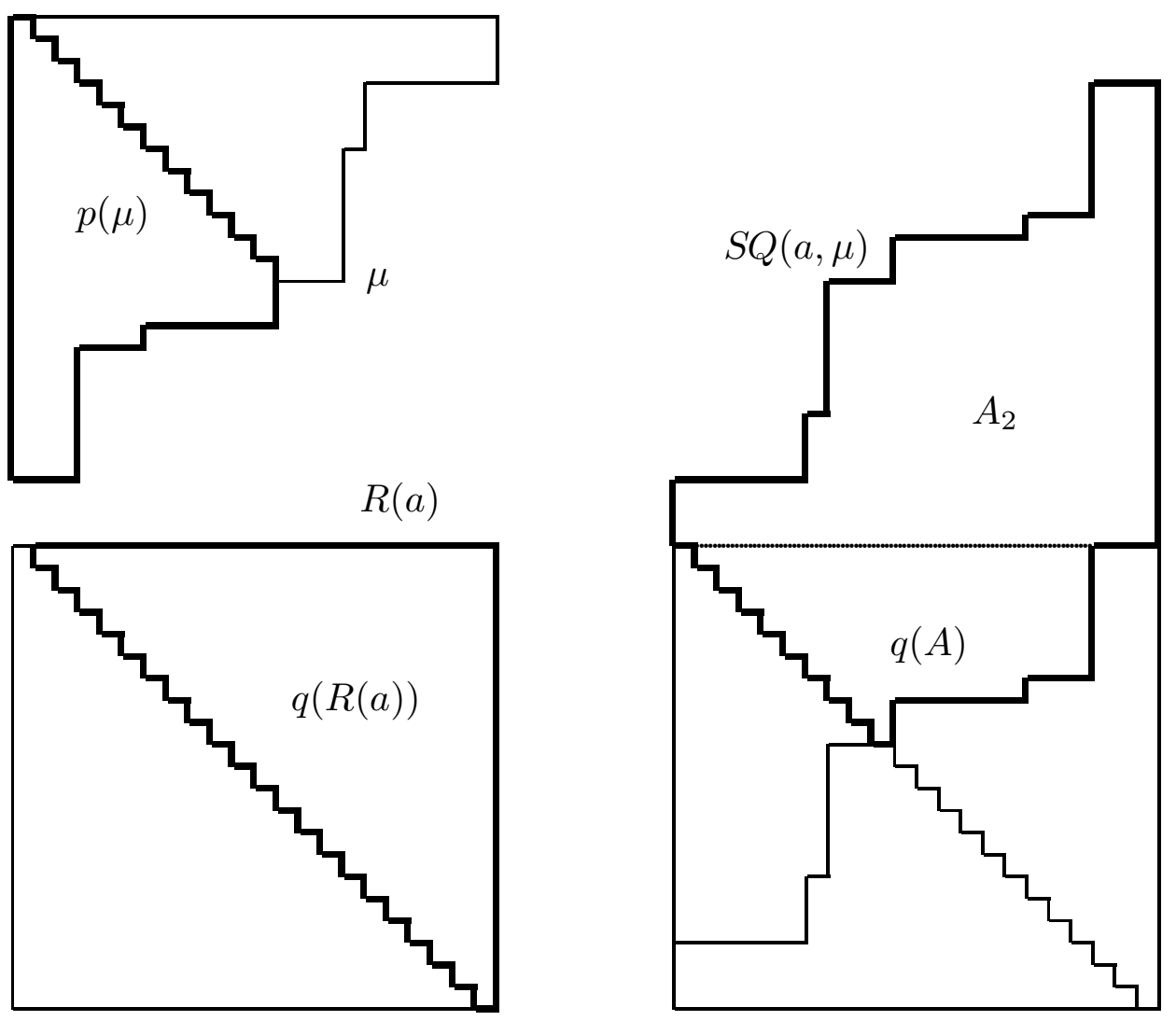

Figure 4

Finally we concern ourselves with Regev's refinement [6] of Theorem 2 for partitions $\mu$ of the form $\mu=\left(\lambda_{1}, \ldots, \lambda_{s} \mid \lambda_{1}-1, \ldots, \lambda_{s}-1\right)$ (in Frobenius notation; cf. [3, p. 3]), where $\lambda_{1}>\cdots>\lambda_{s}>0$. Given such a partition $\mu$, we split it into two "halves" by cutting it along the diagonal as is illustrated in the top-left part of Figure 4 in an example where $\mu=\left(22^{3}, 16^{3}, 15^{6}, 12^{2}, 6,3^{6}\right)=(21,20,19,12,11,10,8,7,6,5,4,3$ | $20,19,18,11,10,9,7,6,5,4,3,2)$. Denote the lower-left region by $p(\mu)$. Given $a \geq$ $\mu_{1}-1=\lambda_{1}$, let $R(a)$ denote the $a \times(a+1)$ rectangle $R_{a, a+1}=\left((a+1)^{a}\right)$. Again, we split it into two halves, by cutting it along the diagonal as is illustrated in the bottom-left part of Figure 4 for $a=21$. Denote the upper-right region by $q(R(a))$. Now consider $S Q(a, \mu):=S Q(a, a+1, \mu)$. Recall that this region consists of the $a \times(a+1)$ rectangle $R_{a, a+1}$, of which a rotated copy of $\mu$ has been removed from its bottom-right corner, and on top of which and to the left of which was placed a rotated copy of $\mu$ each. Once again we split it into two halves along the diagonal of 
the rectangle $R_{a, a+1}$, as is illustrated in Figure 4. (There, we have chosen $a=21$ and $\mu=\left(22^{3}, 16^{3}, 15^{6}, 12^{2}, 6,3^{6}\right)$. Since it is of no relevance for us here, we have omitted to display the rotated copy of $\mu$ placed to the left of the rectangle $R_{21,22}$.) The part of $S Q(a, \mu)$ above the diagonal (in the example of Figure 4 this is the region inside the thick boundary) consists of two parts, the rotated copy of $\mu$ on top, which we denote by $A_{2}$, and the part which remained from $R(a)$, which we denote by $q(A)$.

If $H$ is a subregion of $G$ (an example being $H=p(\mu)$ and $G=\mu$ ), let us write $H P_{G}(H)$ for the multiset of hook pairs of the cells of $H$ measured inside $G$, i.e., arm length and leg length are taken with respect to the boundaries of $G$ (and not with respect to the boundaries of $H$ ). (See [6, Sec. 1] for an elaborate example. The definition is motivated by the definition of shifted hook length when $p(\mu)$ is regarded as a shifted partition, cf. [3, Ch. III, Sec. 8, Ex. 12].)

With this notation, the main result from [6, Theorem I, (I.1)] reads as follows.

Theorem 3. With the assumptions and notations as explained above, the following identity holds between multisets of hook pairs:

$$
H P_{\mu}(p(\mu)) \cup H P_{R(a)}(q(R(a)))=H P_{S Q(a, \mu)}(q(A)) \cup H P_{S Q(a, \mu)}\left(A_{2}\right) .
$$

3. The "master bijection" - Proof of Theorem 1. It is obvious that for a proof of Theorem 1 it suffices to consider just those cells $c$ of $S R_{n, k}(\mu)$ and $S R_{n, k}(\widetilde{\mu})$ for which the corresponding hook pairs $(a(c), l(c))$ have a fixed arm length, $a(c)=d$ say, and to show that the multiset of leg lengths of these cells in $S R_{n, k}(\mu)$ agrees with the multiset of leg lengths of these cells in $S R_{n, k}(\widetilde{\mu})$. To illustrate what we mean, choose $n=10, k=8, \mu=(7,7,5,4,4,3,3,1)$. The skew diagrams $S R_{n, k}(\mu)$ and $S R_{n, k}(\widetilde{\mu})$ with this choice of parameters are displayed in Figure 5. The Figure also shows the cells in $S R_{n, k}(\mu)$ and $S R_{n, k}(\widetilde{\mu})$ which have arm length $d=2$. The numbers inside the cells are the corresponding leg lengths. We call the cells of a region $R$ which have arm length $d$, the broken column of $R$ in distance $d$. We have to show that, in general, for any $d$ the multiset of leg lengths of cells in the broken column of $S R_{n, k}(\mu)$ in distance $d$ is equal to the multiset of leg lengths of cells in the broken column of $S R_{n, k}(\widetilde{\mu})$ in distance $d$.

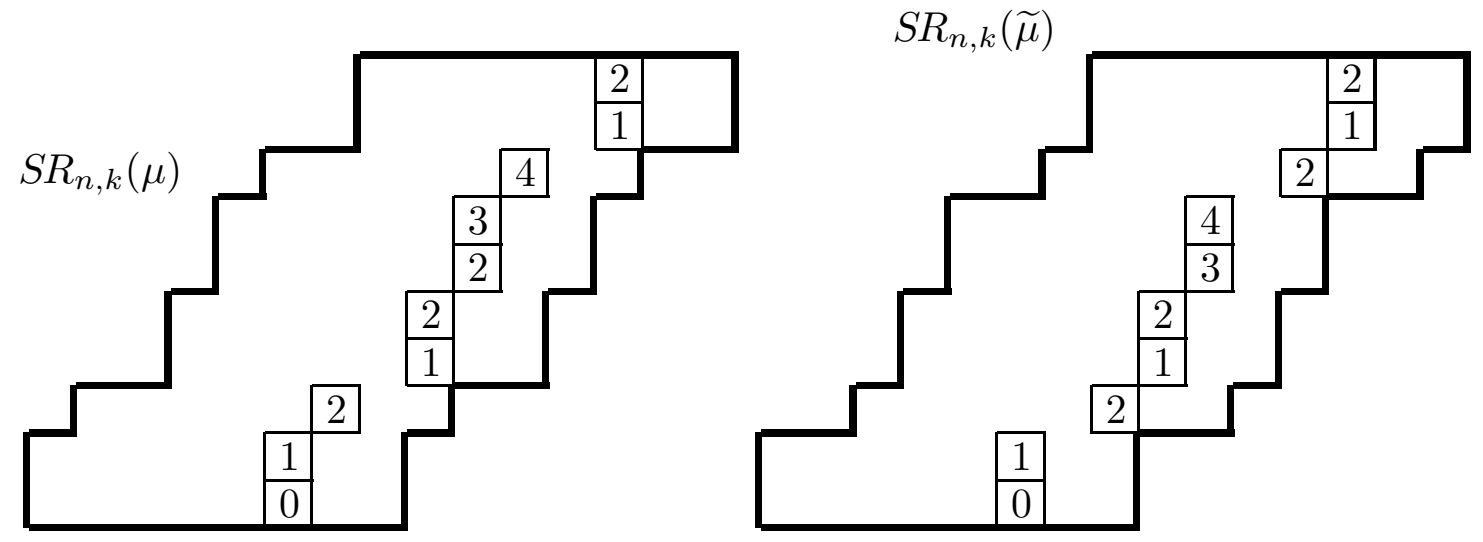

Figure 5 
If we rotate $S R_{n, k}(\widetilde{\mu})$ by $180^{\circ}$, then $S R_{n, k}(\mu)$ and the rotated $S R_{n, k}(\widetilde{\mu})$ fit together side by side. Figure 6 shows the result in the case of our example of Figure 5. The numbers in the broken column which is to the left of the staircase that forms the border between the two regions are the vertical distances of the cells to the "bottom" of the diagram (consisting of the staircase and the base line to the left of the staircase), while the numbers in the broken column which is to the right of the staircase are the vertical distances of the cells to the "top" of the diagram (consisting of the staircase and the top line to the right of the staircase). Our task is to set up a bijection between these two multisets of numbers.

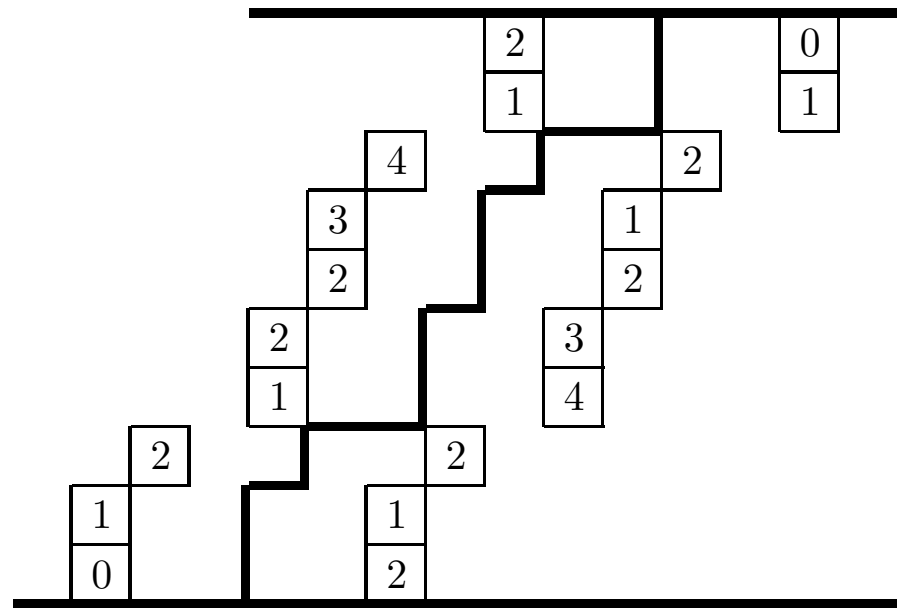

Figure 6

Lemma. Let $S$ be a given staircase (see Figure 6). Let $C_{l}$ be the broken column in distance $d$ left of $S$, and let $C_{r}$ be the broken column in distance $d$ right of $S$. Then there is an explicit bijection (the "master bijection") between the multiset $\{l(c): c \in$ $\left.C_{l}\right\}$ and the multiset $\left\{l^{\prime}(c): c \in C_{r}\right\}$. Here, $l(c)$ denotes the distance of cell $c$ to the bottom of the diagram, and $l^{\prime}(c)$ denotes the distance of cell $c$ to the top of the diagram.

Proof. We claim that the following algorithm defines such a bijection:

(MB1) Read the numbers in the cells of $C_{l}$ one after the other by considering the cells in the order bottom to top. While reading, out of each maximal increasing subsequence of numbers form a stack. Place the stacks in such a way that numbers of the same size are at the same height. (In our example in Figure 6, the numbers $0,1,2,1,2,2,3,4,1,2$ are read in. See the left part of Figure 7 for the result after dividing this sequence into stacks.)

(MB2) Move all the numbers of the first stack which are smaller than the numbers in the second stack to the second stack. Repeat this procedure with the second and third stack, etc. (Thus, in our example in the left part of Figure 7 we would move 0 from the first to the second stack, 0,1 from the second to the 
THE Electronic journal of Combinatorics 7 (2000), \#R27

third, and 0 from the third to the fourth stack. The result is displayed in the right part of Figure 7 .)

(MB3) Read the numbers in the result in the order from the last stack to the first, and in each stack from bottom to top. (Thus, from the right part of Figure 7 we would read $0,1,2,1,2,3,4,2,1,2$.)

The claim is that the output of this algorithm is the numbers in the cells of $C_{r}$ read by considering the cells in the order top to bottom (which for our running example can be verified in Figure 6). Clearly, this would immediately prove the Lemma because it is obvious how to invert the algorithm.

$\begin{array}{llllllll} & & 4 & & & & 4 & \\ 2 & 2 & 2 & 2 & & & 3 & \\ 1 & 1 & & 1 & 2 & 2 & 2 & 2 \\ 0 & & & & 1 & & 1 & 1 \\ 0 & & & & & & & \\ & & & & & & \end{array}$

Figure 7

In order to prove the claim, we first introduce some notation. The staircase $S$ consists of, alternately, vertical and horizontal pieces. Let the lengths of these pieces be $v_{1}, h_{1}, v_{2}, h_{2}, \ldots, v_{p-1}, h_{p-1}, v_{p}$, where $v_{i}$ stands for the length of the $i$-th vertical piece, if counted from bottom to top, and where $h_{i}$ stands for the length of the $i$-th horizontal piece. In the example in Figure 6 we have $v_{1}=2, h_{1}=1, v_{2}=1, h_{2}=2$, $v_{3}=2, h_{3}=1, v_{4}=2, h_{4}=1, v_{5}=1, h_{5}=2, v_{6}=2$.

Furthermore, let the maximal increasing subsequences when reading the numbers from $C_{l}$ (from bottom to top) be $0,1, \ldots, M_{1} ; m_{2}, m_{2}+1, \ldots, M_{2} ; \ldots ; m_{q}, m_{q}+$ $1, \ldots, M_{q}$, so that the corresponding diagram in the style of Figure 7 looks like

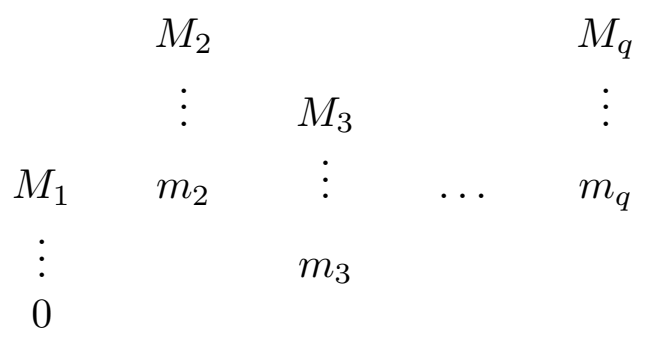

Then there exist uniquely determined integers $i_{1}, \ldots, i_{q}$ and $j_{1}, \ldots, j_{q}$ with $1 \leq i_{1}<$ $\cdots<i_{q}=p, 1=j_{1}<\cdots<j_{q} \leq p$, and $j_{t} \leq i_{t}$ for all $t$, such that

$$
\begin{aligned}
& M_{1}=v_{j_{1}}+\cdots+v_{i_{1}}-1, \quad h_{j_{1}}+\cdots+h_{i_{1}-1} \leq d \quad \text { and } \quad h_{j_{1}}+\cdots+h_{i_{1}}>d \text {, } \\
& m_{2}=v_{j_{2}}+\cdots+v_{i_{1}}, \quad h_{j_{2}-1}+\cdots+h_{i_{1}}>d \quad \text { and } \quad h_{j_{2}}+\cdots+h_{i_{1}} \leq d, \\
& M_{2}=v_{j_{2}}+\cdots+v_{i_{2}}-1, \quad h_{j_{2}}+\cdots+h_{i_{2}-1} \leq d \quad \text { and } \quad h_{j_{2}}+\cdots+h_{i_{2}}>d,(1) \\
& m_{q}=v_{j_{q}}+\cdots+v_{i_{q-1}}, \quad h_{j_{q}-1}+\cdots+h_{i_{q-1}}>d \quad \text { and } \quad h_{j_{q}}+\cdots+h_{i_{q-1}} \leq d, \\
& M_{q}=v_{j_{q}}+\cdots+v_{i_{q}}-1, \quad h_{j_{q}}+\cdots+h_{i_{q}-1} \leq d \text {. }
\end{aligned}
$$


Similarly, let the maximal increasing subsequences when reading the numbers from $C_{r}$ (from top to bottom) be $0,1, \ldots, \tilde{M}_{q} ; \ldots ; \tilde{m}_{2}, \tilde{m}_{2}+1, \ldots, \tilde{M}_{2} ; \tilde{m}_{1}, \tilde{m}_{1}+1, \ldots, \tilde{M}_{1}$, so that the corresponding diagram in the style of Figure 7 looks like

$\begin{array}{ccccc} & \tilde{M}_{2} & & & \tilde{M}_{q} \\ \tilde{M}_{1} & \vdots & & \tilde{M}_{q-1} & \vdots \\ \vdots & \tilde{m}_{2} & \ldots & \vdots & \vdots \\ \tilde{m}_{1} & & & \tilde{m}_{q-1} & \vdots \\ & & & & 0\end{array}$

Then there exist uniquely determined integers $\tilde{j}_{1}, \ldots, \tilde{j}_{q}$ and $\tilde{i}_{1}, \ldots, \tilde{i}_{q}$ with $1 \leq \tilde{j}_{1}<$ $\cdots<\tilde{j}_{q}=p, 1=\tilde{i}_{1}<\cdots<\tilde{i}_{q} \leq p$, and $\tilde{j}_{t} \geq \tilde{i}_{t}$ for all $t$, such that

$$
\begin{aligned}
& \tilde{M}_{q}=v_{\tilde{j}_{q}}+\cdots+v_{\tilde{i}_{q}}-1, \\
& h_{\tilde{j}_{q}-1}+\cdots+h_{\tilde{i}_{q}} \leq d \quad \text { and } \quad h_{\tilde{j}_{q}-1}+\cdots+h_{\tilde{i}_{q}-1}>d, \\
& \tilde{m}_{q-1}=v_{\tilde{j}_{q-1}}+\cdots+v_{\tilde{i}_{q}} \\
& h_{\tilde{j}_{q-1}}+\cdots+h_{\tilde{i}_{q}-1}>d \quad \text { and } \quad h_{\tilde{j}_{q-1}-1}+\cdots+h_{\tilde{i}_{q}-1} \leq d, \\
& \tilde{M}_{q-1}=v_{\tilde{j}_{q-1}}+\cdots+v_{\tilde{i}_{q-1}}-1 \text {, } \\
& h_{\tilde{j}_{q-1}-1}+\cdots+h_{\tilde{i}_{q-1}} \leq d \quad \text { and } \quad h_{\tilde{j}_{q-1}-1}+\cdots+h_{\tilde{i}_{q-1}-1}>d \text {, } \\
& \tilde{m}_{1}=v_{\tilde{j}_{1}}+\cdots+v_{\tilde{i}_{2}}, \\
& h_{\tilde{j}_{1}}+\cdots+h_{\tilde{i}_{2}-1}>d \quad \text { and } \quad h_{\tilde{j}_{1}-1}+\cdots+h_{\tilde{i}_{2}-1} \leq d, \\
& \tilde{M}_{1}=v_{\tilde{j}_{1}}+\cdots+v_{\tilde{i}_{1}}-1, \quad h_{\tilde{j}_{1}-1}+\cdots+h_{\tilde{i}_{1}} \leq d .
\end{aligned}
$$

The claim is equivalent to the assertion that $\tilde{M}_{t}=M_{t}$ and $\tilde{m}_{t}=m_{t+1}$ for all $t$. In view of (1) and (2), this would immediately follow once we show that $\tilde{j}_{t}=i_{t}$ and $\tilde{i}_{t}=j_{t}$ for all $t$. In order to do that, it suffices to derive the inequalities in (2) from those of (1). Indeed, the general form of the inequalities in (1) is

$$
\begin{aligned}
& h_{j_{t+1}-1}+\cdots+h_{i_{t}}>d \text { and } h_{j_{t+1}}+\cdots+h_{i_{t}} \leq d \text {, } \\
& h_{j_{t}}+\cdots+h_{i_{t}-1} \leq d \quad \text { and } \quad h_{j_{t}}+\cdots+h_{i_{t}}>d .
\end{aligned}
$$

In particular, the first inequality in (3) implies that

$$
h_{j_{t+1}-1}+\cdots+h_{i_{t+1}-1}>d,
$$

since by assumption we have $i_{t} \leq i_{t+1}-1$. Similarly, the first inequality in (4) implies that

$$
h_{j_{t+1}-1}+\cdots+h_{i_{t}-1} \leq d,
$$

since by assumption we have $j_{t} \leq j_{t+1}-1$. Altogether, the inequalities in (3)-(6) cover those of (2) with $\tilde{j}_{t}=i_{t}$ and $\tilde{i}_{t}=j_{t}$.

This concludes the proof of the Lemma. 
4. Proof of Theorem 2. The preceding bijection allows us to construct a bijection for Theorem 2. We have to set up a bijection between the hook pairs in $R_{n, k} \cup \mu$ and the hook pairs in $S Q(n, k, \mu)$.

To begin with, we identify the hook pairs in a subregion of $R_{n, k}$ with the hook pairs in a subregion of $S Q(n, k, \mu)$. The subregion of $R_{n, k}, S_{1}$ say, consists of the first $k-\mu_{1}$ columns of $R_{n, k}$ together with a copy of $\mu$, reflected upside down, on the bottom of $R_{n, k}$ (see the left part of Figure 8 for an example with $n=18, k=24$, $\left.\mu=\left(15^{3}, 6^{3}, 3^{6}\right)\right)$. The subregion of $S Q(n, k, \mu), S_{2}$ say, consists of the (rotated) copy of $\mu$ on the left of $S Q(n, k, \mu)$, together with the next $k-\mu_{1}$ columns of $S Q(n, k, \mu)$ (see the right part of Figure 8).
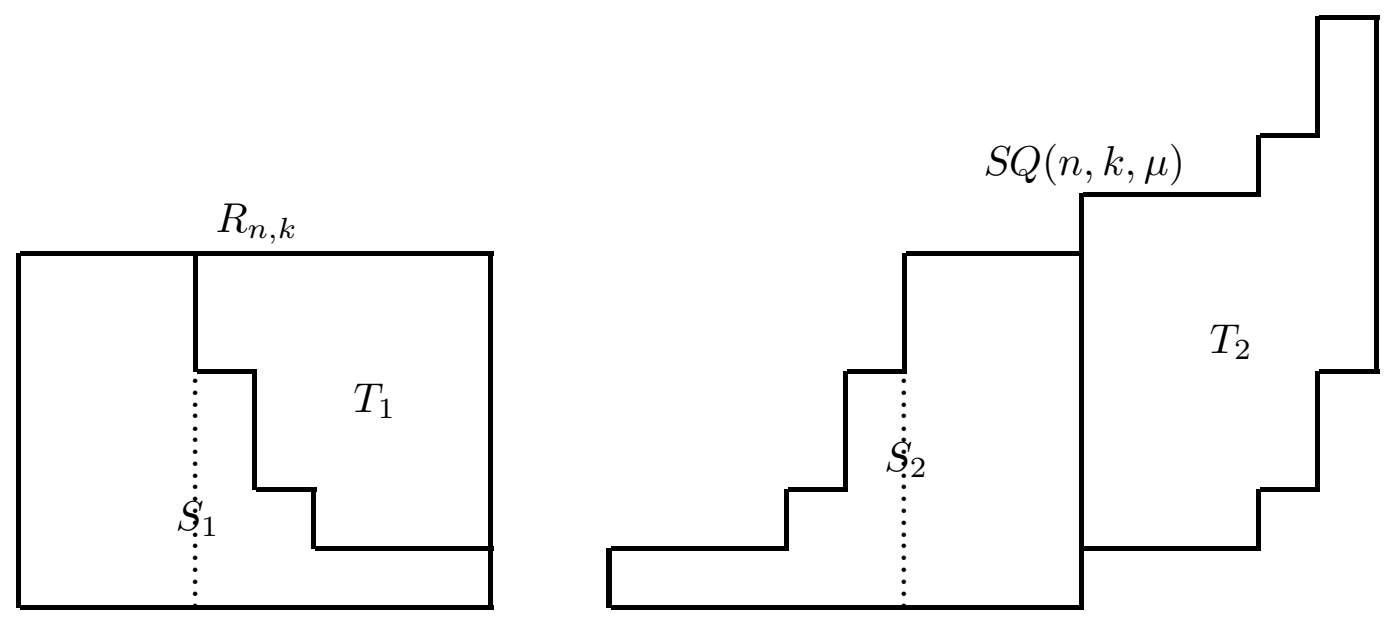

Figure 8

It is completely obvious that, row-wise, the hook pairs in these subregions must be the same. I.e., reading hook pairs from left to right in the first row of $S_{1}$ gives exactly the same as reading hook pairs from left to right in the first row of $S_{2}$, the same being true for the second rows, etc.

Therefore, what remains is to identify the hook pairs in the remaining regions. Let us denote the complement of $S_{1}$ in $R_{n, k}$ by $T_{1}$, and the complement of $S_{2}$ in $S Q(n, k, \mu)$ by $T_{2}$ (see Figure 8 ). Then we have to identify the hook pairs in $T_{1} \cup \mu$ with the hook pairs in $T_{2}$.

Let us again consider broken columns in some given distance $d$ from the right boundaries. This is indicated in Figure 9. (In this example, $n=18, k=24, \mu=$ $\left(15^{3}, 6^{3}, 3^{6}\right)$, and $d=4$.) The figure also shows "fake" parts of broken columns for $\mu$ and $T_{2}$, i.e., parts of broken columns which lie outside of the regions. They are shown with dotted surroundings and should be ignored for the moment. We have to identify the multiset of leg lengths in the broken columns of $\mu$ and $T_{1}$ (excluding the "fake" part) with the multiset of leg lengths in the broken column of $T_{2}$ (excluding the "fake" part). Let us denote the multiset of leg lengths in the broken column of $\mu$ 

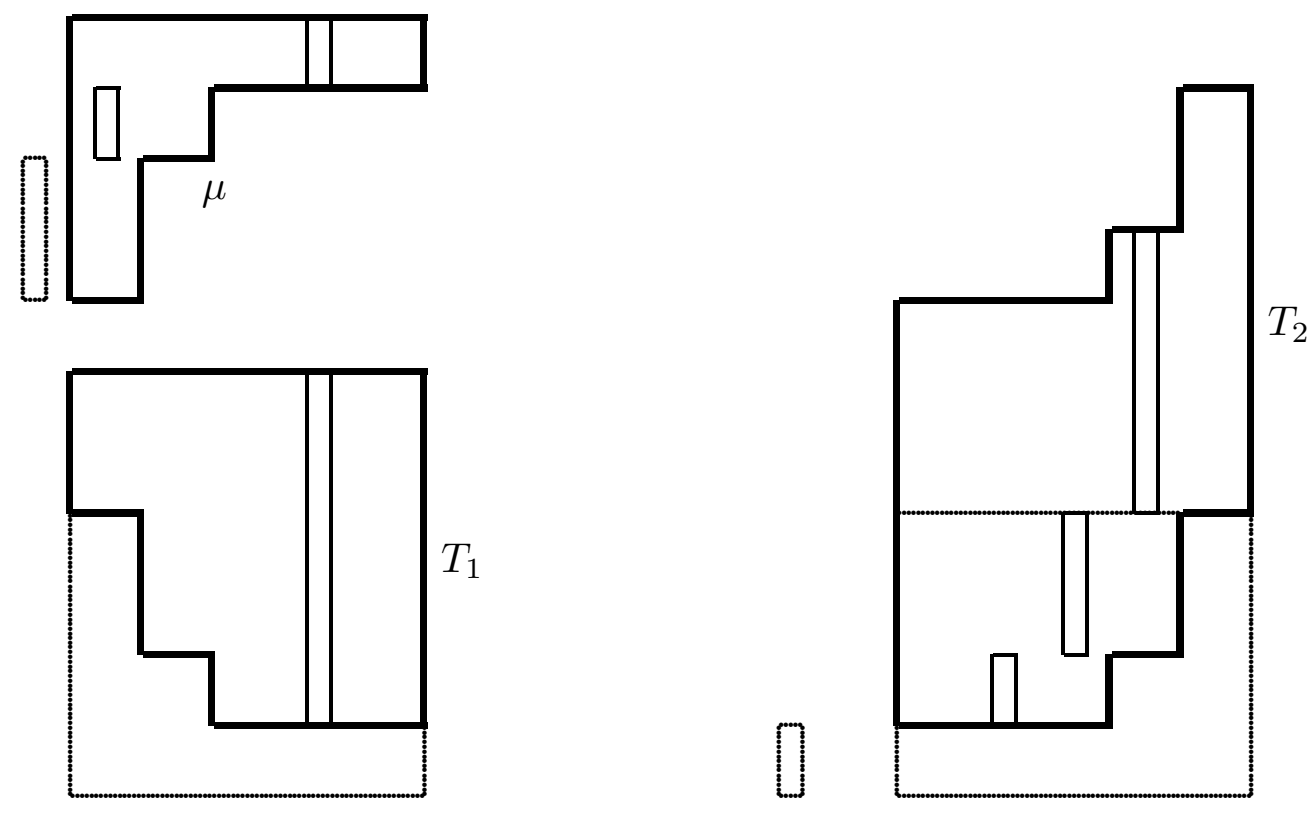

Figure 9

by $L(\mu ; d)$, and similarly for $T_{1}$ and $T_{2}$.

In order to accomplish this identification, we observe that the Lemma from Section 3 provides a bijection between the multiset of leg lengths in the broken column of $\mu$, the "fake" part included, and the part of the broken column of $T_{2}$ which is to the left of the (rotated) copy of $\mu$ that has been removed from $S Q(n, k, \mu)$ (indicated by dotted lines in Figure 9), again the "fake" part included. (In Figure 9, this part of the broken column is the one below the dotted horizontal line running through $T_{2}$, the "fake" part included.) Let us denote the former multiset by $L_{f}(\mu ; d)$, and the latter by $L_{f}\left(T_{2} ; d\right)$.

Furthermore, with the notation $[[N]]:=\{0,1, \ldots, N-1\}$, we have

$$
\begin{gathered}
L(\mu ; d) \cup L\left(T_{1} ; d\right)=\left(L_{f}(\mu ; d) \backslash\left[\left[\ell(\mu)+1-\min \left\{k: \mu_{k} \leq d\right\}\right]\right]\right) \\
\cup\left([[n]] \backslash\left[\left[\max \left\{k: \mu_{k} \geq \mu_{1}-d\right\}\right]\right]\right) \\
\quad=\left(L_{f}(\mu ; d) \cup[[n]]\right) \backslash\left(\left[\left[\ell(\mu)+1-\min \left\{k: \mu_{k} \leq d\right\}\right]\right] \cup\left[\left[\max \left\{k: \mu_{k} \geq \mu_{1}-d\right\}\right]\right]\right),
\end{gathered}
$$

where $\ell(\mu)$ denotes the number of parts of $\mu$ (equivalently, the number of rows of $\mu$ ), and

$$
\begin{aligned}
& L\left(T_{2} ; d\right)=\left(L_{f}\left(T_{2} ; d\right) \backslash\left[\left[\max \left\{k: \mu_{k}+d \geq \mu_{1}\right\}\right]\right]\right) \\
& \cup\left([[n]] \backslash\left[\left[\ell(\mu)+1-\min \left\{k: \mu_{k} \leq d\right\}\right]\right]\right) \\
& =\left(L_{f}\left(T_{2} ; d\right) \cup[[n]]\right) \backslash\left(\left[\left[\max \left\{k: \mu_{k}+d \geq \mu_{1}\right\}\right]\right] \cup\left[\left[\ell(\mu)+1-\min \left\{k: \mu_{k} \leq d\right\}\right]\right]\right) .
\end{aligned}
$$


In view of our previous observation that the multisets $L_{f}(\mu ; d)$ and $L_{f}\left(T_{2} ; d\right)$ are in bijection, it is now completely evident that the multisets $L(\mu ; d) \cup L\left(T_{1} ; d\right)$ and $L\left(T_{2} ; d\right)$ agree.

Remark. The argument thus far was not completely bijective. But it could easily be made bijective, for example, using the simplified version of the involution principle described in [9, bottom of p. 80ff]. There are other possibilities, but none of these seem to yield naturally defined mappings. In particular, these would not add any further insight. It is the "master bijection" from Section 3 and the computations in (7) and (8) which really explain why Theorem 2 is true.

5. Proof of Theorem 3. In a similar manner, Theorem 3 can be proved. We have to prove that the hook pairs in $p(\mu)$ and $q(R(a))$ (measured inside $\mu$ and $R(a)$, respectively) are the same as the hook pairs in $q(A)$ and $A_{2}$ (measured inside $S Q(a, \mu)$ ), see Figure 4.

Again, it suffices to consider a broken column in some distance $d$ from the right boundaries in the corresponding figures, and to show that the leg lengths along the broken columns agree (see Figure 10, where $d=4$; the "fake" part of the broken column in $p(\mu)$ should be ignored for the moment), i.e.,

$$
L(p(\mu) ; d) \cup L(q(R(a)) ; d)=L(q(A) ; d) \cup L\left(A_{2} ; d\right),
$$

using the same notation as before.

Again, the key is the Lemma from Section 3. What Figure 11 shows is obtained by the following construction: In Figure 10, in the part which shows $p(\mu)$, the extremal point, denoted by $M$, on the "diagonal" is circled. The corresponding point, denoted by $M^{\prime}$, in the part which shows $S Q(a, \mu)$ is also circled. The top-most part of the broken column in $p(\mu)$ reaches a certain height, $h$ say, above $M$. (The height $h$ can be any nonnegative integer.) In Figure 10, we have $h=2$. Now cut the Figure which represents $S Q(a, \mu)$ by the horizontal line which is $h$ units below $M^{\prime}$. In addition, cut off $A_{2}$. This gives Figure 11 (where $h=2$ ), upon also adding a broken column in distance $d$ to the right of the path which runs from bottom-left to top-right, and upon completing a broken column in distance $d$ to the left of the path.

Let us denote the leg lengths (measured "to the bottom," i.e., with respect to the path which runs from bottom-left to top-right) in the broken column to the left by $L_{f}(q(A) ; d)$. These leg lengths contain all those which appear in $L(q(A) ; d)$, plus the additional ones in the added part of the broken column. More precisely, it is not difficult to see that (at this point the special form of $\mu$ enters)

$$
L_{f}(q(A) ; d)=L(q(A) ; d) \cup[[d]] .
$$

Furthermore, the broken column to the right of the path is in perfect correspondence with the broken column of $p(\mu)$ (with the "fake" part included; compare Figure 10), which is seen by rotation by $180^{\circ}$. Let us denote the leg lengths (measured "to the top," i.e., with respect to the path which runs from bottom-left to top-right) in the broken column to the right by $L_{f}(p(\mu) ; d)$. Obviously we have

$$
L_{f}(p(\mu) ; d)=L(p(\mu) ; d) \cup\left[\left[\ell(\mu)+1-\min \left\{k: \mu_{k} \leq d\right\}\right]\right] .
$$



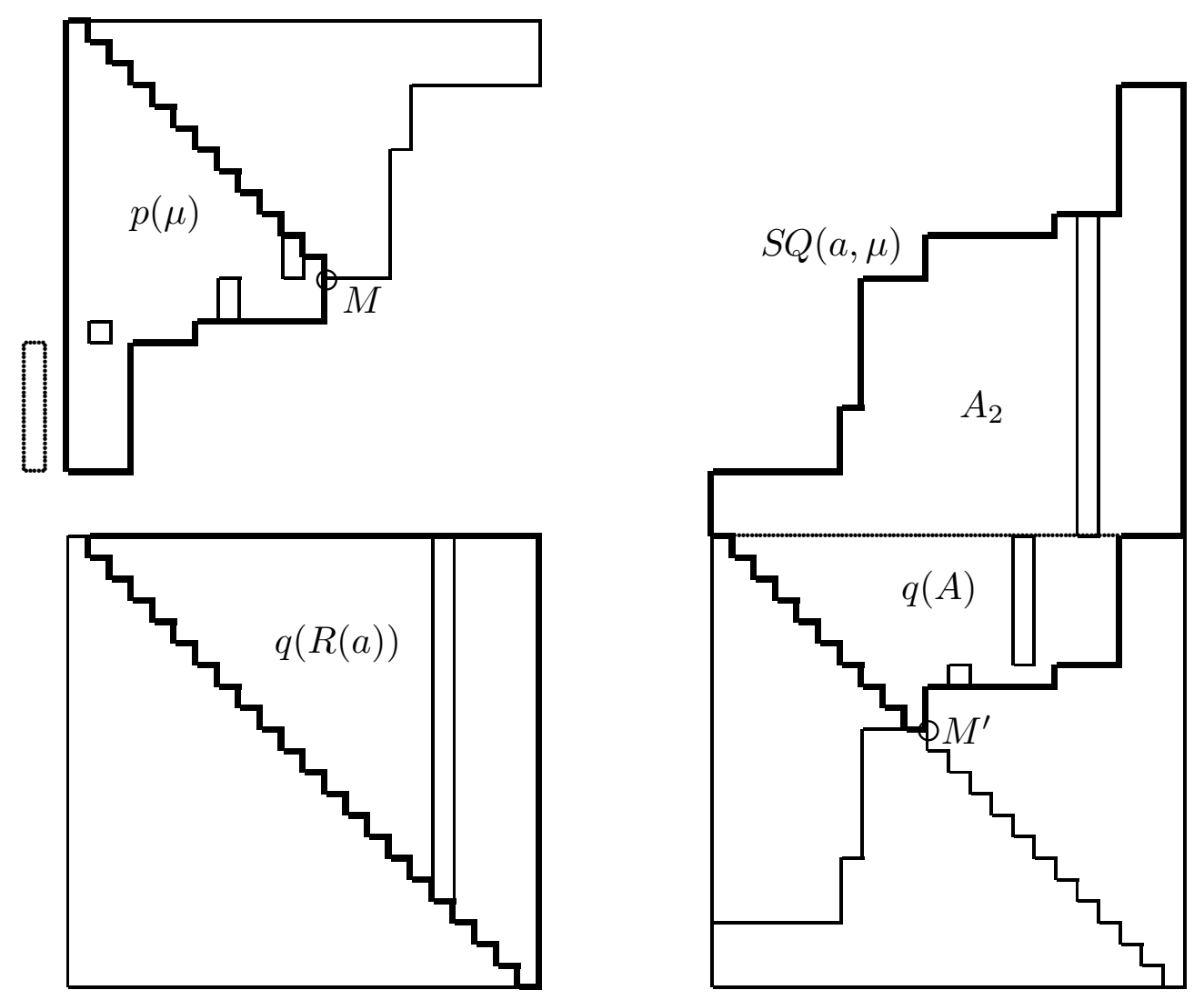

Figure 10

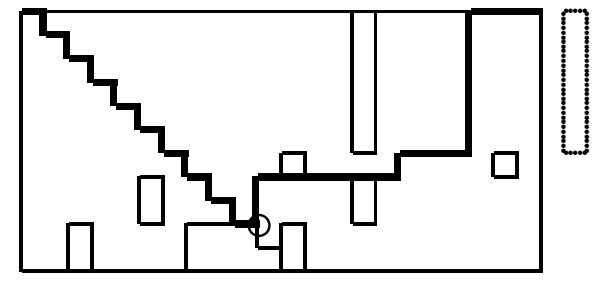

Figure 11

The point of the construction in Figure 11 is that the Lemma from Section 3 tells us that $L_{f}(q(A) ; d)=L_{f}(p(\mu) ; d)$. Now, if we combine everything then we are done: We have

$$
\begin{aligned}
L(p(\mu) ; d) \cup & L(q(R(a)) ; d) \\
& =\left(L_{f}(p(\mu) ; d) \backslash\left[\left[\ell(\mu)+1-\min \left\{k: \mu_{k} \leq d\right\}\right]\right]\right) \cup([[a]] \backslash[[d]]) \\
& =\left(L_{f}(p(\mu) ; d) \cup[[a]]\right) \backslash\left(\left[\left[\ell(\mu)+1-\min \left\{k: \mu_{k} \leq d\right\}\right]\right] \cup[[d]]\right)
\end{aligned}
$$


and

$$
\begin{gathered}
L(q(A) ; d) \cup L\left(A_{2} ; d\right)=\left(L_{f}(q(A) ; d) \backslash[[d]]\right) \cup\left([[a]] \backslash\left[\left[\ell(\mu)+1-\min \left\{k: \mu_{k} \leq d\right\}\right]\right]\right) \\
=\left(L_{f}(q(A) ; d) \cup[[a]]\right) \backslash\left([[d]] \cup\left[\left[\ell(\mu)+1-\min \left\{k: \mu_{k} \leq d\right\}\right]\right]\right) .
\end{gathered}
$$

Clearly, in view of $L_{f}(q(A) ; d)=L_{f}(p(\mu) ; d)$ these two expressions agree, as desired. Hence, the assertion of Theorem 3 is established.

Remark. Again, the above argument was not completely bijective. Statements analogous to those in the Remark at the end of Section 4 apply also here.

\section{REFERENCES}

1. C. Bessenrodt, On hooks of Young diagrams, Ann. Combin. 2 (1998), 103-110.

2. S. Janson, Hook lengths in a skew Young diagram, Electron. J. Combin. 4 (1997), paper \#R24.

3. I. G. Macdonald, Symmetric Functions and Hall Polynomials, second edition, Oxford University Press, New York/London, 1995.

4. A. Postnikov and A. Regev, Hook and content number identities, preprint.

5. A. Regev, Generalized hook and content numbers identities, Europ. J. Combin. (to appear).

6. A. Regev, Generalized hook and content numbers identities - the projective case, Europ. J. Combin. (to appear).

7. A. Regev and A. Vershik, Asymptotics of Young diagrams and hook numbers, Electron. J. Combin. 4 (1997), paper \#R22.

8. A. Regev and D. Zeilberger, Proof of a conjecture on multisets of hook numbers, Ann. Combin. 1 (1997), 391-394.

9. R. P. Stanley, Enumerative Combinatorics, Vol. 1, Wadsworth \& Brooks/Cole, Pacific Grove, California, 1986, reprinted by Cambridge University Press, Cambridge, 1998.

Institut für Mathematik der Universität Wien, Strudlhofgasse 4, A-1090 Wien, Austria. 